\section{CABG surgery does not increase the risk of late cognitive decline}

Several studies have indicated that delayed effects of coronary artery bypass grafting (CABG) surgery might contribute to cognitive decline and neurological complications in some individuals. Most studies, however, have not compared cognitive decline in patients undergoing surgery with that in suitable control patients, making it difficult to establish the causes of cognitive decline in this patient population. To address this issue, Selnes and colleagues investigated the long-term outcomes of patients with coronary artery disease who received either CABG surgery or nonsurgical intervention.

A total of 152 patients who underwent CABG surgery and 92 demographically and medically similar patients with coronary artery disease who were not scheduled to undergo surgical intervention (the control group) were enrolled in this 6-year study. Memory and cognitive tests (including visual and verbal memory, motor speed, and attention domains) were performed at enrollment and after 3, 12, 36 and 72 months; 96 patients who had undergone surgery and 61 control patients completed 6 years of followup. No significant differences were observed in cognitive scores between the two groups at baseline and throughout the study period, although mild, late cognitive decline occurred in both groups during follow-up.

These findings argue against a link between CABG surgery and late cognitive decline ( $\geq 5$ years after surgery). The authors suggest that normal aging processes or progression of vascular disease during the 6 years of followup might be responsible for the mild cognitive decline observed in the study.

Original article Selnes OA et al. (2008) Cognition 6 years after surgical or medical therapy for coronary artery disease. Ann Neurol 63: 581-590

\section{Mutations in protocadherin gene lead to female epilepsy and mental retardation}

Epilepsy and mental retardation limited to females (EFMR) is an X-linked disorder characterized by an unusual inheritance pattern in which carrier women develop seizures and cognitive impairment in infancy and early childhood, whereas transmitting men are spared. To examine the genetic basis of EFMR, Dibbens et al. studied the gene sequences of members of seven families with a history of this disease.

By resequencing $737 \mathrm{X}$-chromosome genes from members of these families, the authors identified seven loss-of-function mutations in the protocadherin 19 (PCDH19) gene that were unique for each family and were all associated with EFMR. In five families, the mutations resulted in the introduction of a premature termination codon into the $\mathrm{PCDH} 19$ messenger RNA, which, in two families, was found to result in messenger RNA degradation via nonsensemediated decay surveillance complexes. The remaining two families were found to have missense mutations, which the authors suggest lead to a loss of $\mathrm{PCDH19}$ function through impaired calcium binding. The authors showed that $\mathrm{PCDH} 19$ is expressed in the developing cortical plate, the lateral nuclei of the amygdala, the subcortical regions and the ganglionic eminence, which is consistent with the disease phenotype.

The cadherins are known to have important roles in brain development and function, but this is the first time that epilepsy and mental retardation has been directly linked to mutations in a member of the cadherin gene superfamily. The mechanism underlying the unusual inheritance pattern of EFMR remains unclear, but the authors hypothesize that it might be attributable to functional rescue by $P C D H 11 Y$, a related but nonparalogous protocadherin gene on the Y chromosome.

Original article Dibbens LM et al. (2008) X-linked protocadherin 19 mutations cause female-limited epilepsy and cognitive impairment. Nat Genet 40: 776-781

\section{Repetitive magnetic stimulation shows promise in rehabilitation after pediatric stroke}

Randomized trials of repetitive transcranial magnetic stimulation (rTMS) in adults have shown that this technique can improve motor function after subcortical stroke. To determine whether rTMS could provide similar benefits to children with disabling hemiparesis following arterial ischemic stroke, Kirton et al. conducted a randomized study comprising 10 children 